\title{
The quantity of quantum information and its metaphysics
}

\author{
Vasil Penchev $^{1}$
}

\begin{abstract}
The quantum information introduced by quantum mechanics is equivalent to that generalization of the classical information from finite to infinite series or collections. The quantity of information is the quantity of choices measured in the units of elementary choice. The qubit can be interpreted as that generalization of bit, which is a choice among a continuum of alternatives. The axiom of choice is necessary for quantum information. The coherent state is transformed into a wellordered series of results in time after measurement. The quantity of quantum information is the ordinal corresponding to the infinity series in question. Number and being (by the meditation of time), the natural and artificial turn out to be not more than different hypostases of a single common essence. This implies some kind of neo-Pythagorean ontology making related mathematics, physics, and technics immediately, by an explicit mathematical structure.
\end{abstract}

\section{INTRODUCTION}

The conception of quantum information was introduced in the theory of quantum information studying the phenomena of entanglement in quantum mechanics. The entanglement was theoretically forecast in the famous papers of Einstein, Podolsky, and Rosen (1935) and independently by Shrödinger (1935) deducing it from Hilbert space, the basic mathematical formalism of quantum mechanics. However, the former three demonstrated the forecast phenomenon as the proof of the alleged "incompleteness of quantum mechanics". John Bell (1964) deduced a sufficient condition as an experimentally verifiable criterion in order to distinguish classical from quantum correlation (entanglement). Aspect, Grangier, and Roger (1981, 1982) confirmed experimentally the existence of quantum correlations exceeding the upper limit of the possible classical correlations. The theory of quantum information has thrived since the end of the last century in the areas of quantum computer, quantum communication, and quantum cryptography.

The unit of quantum information is the 'quantum bit', "qubit" definable as the normed superposition of any two orthogonal subspaces of complex Hilbert space as follows:

'Qubit' is: $\alpha|0\rangle+\beta|1\rangle$ where $\alpha, \beta$ are complex numbers such that, $|\alpha|^{2}+|\beta|^{2}=1$ and $|0\rangle,|1\rangle$ are any two orthonormal vectors (e.g. the orthonormal bases of any two subspaces) in any vector space (e.g. Hilbert space, Euclidean space, etc.). Thus Hilbert space underlying quantum mechanics is representable as the quantity of quantum information and any wave function, i.e. any state of any quantum system being a point in it can be seen as a value of that quantity.

\footnotetext{
1 Dept. of Logical Systems and Models, Institute of Philosophy and Sociology, Bulgarian Academy of Sciences, vasildinev@gmail.com
}

Consequently all physical processes turn out to be quantuminformational, and nature or the universe is a quantum computer processing quantum information.

The qubit is also isomorphic to a ball in Euclidean space, in which two points are chosen: A qubit is equivalently representable as a unit ball in Euclidean space and two points, the one chosen within the ball, and the other being the orthogonal projection on its surface, i.e. as a mapping of a unit ball onto its surface (or any other unit sphere).

The main statement: Quantum information is equivalent to the generalization of information from finite to infinite series.

It is trivial in the range of the axiom of choice.

The axiom of choice is necessary for quantum information in two ways: (1) in order to guarantee the choice even if any constructive approach to be chosen an element of the continuum does not exist; (2) to equate the definition in terms of Hilbert space and that as a choice among a continuum of alternatives:

Indeed the theorems about the absence of hidden variables in quantum mechanics (Neumann 1932; Kochen, Specker 1968) demonstrate that the mathematical formalism of quantum mechanics implies that no well-ordering of any coherent state might exist before measurement.

However, the same coherent state is transformed into a wellordered series of results in time after measurement. In order to be equated the state before and after measurement, the wellordering theorem equivalent to the axiom of choice is necessary.

The measurement mediating between them should be interpreted as an absolutely random choice of an element of the coherent state, for which no constructive way (equivalent to some "hidden variable") can exist in principle.

Thus the quantity of quantum information can describe uniformly the state before and after measurement (equivalent to a choice among an infinite set).

Therefore Hilbert space can be understood as the free variable of quantum information. Then any wave function, being a given value of it, "bounds" an unorderable and a well-ordered state as the quantity of qubits (i.e. the "infinite choices") necessary for the latter to be obtained from the former.

The quantity of quantum information is the ordinal corresponding to the infinity series in question. Both definitions of "ordinal" (Cantor 1897; Neumann 1923) are applicable as the ordinals are relatively "small". The ordinal number corresponds one-to-one to a coherent state as the one and same quantity of quantum information containing in it.

The paper is organized in the following sections. Section 2 introduces the necessary notions and general viewpoint, in which the main statement should be obvious. Section 3 clears up its interpretation in a few relevant reference frames: set theory, foundation of mathematics, theory of information and quantum mechanics. Section 4 presents the conclusions. 


\section{THE NOTIONS AND GENERAL VIEWPOINT}

One can consider the following concepts as relevant to the focus of the paper: information; quantum information; information as a binary series (Kolmogorov); choice; (quantum) information as the quantity of choices; the axiom of choice; a choice from infinity; Hilbert space; coherent state as an equivalent of a statistical ensemble; and quantum mechanics as a theory of quantum information. These concepts will be discussed in a few more details bellow:

"Information" is a very wide and thus undetermined concept. However, its fruitfulness relies just on this in some degree.

It means some relation between orders or orderings. These relation and orderings can be both qualitative and quantitative. Only the latter case is meant in this paper.

The usual quantitative definitions of information refer it to the some mathematical relation of entropies or as some modification or generalization of the quantity of entropy. Indeed entropy can be interpreted as a measure of disorder therefore as equivalent to the opposite of the measure of order as what information is considered.

Thus the quantity of information turns out to refer to some statistical ensembles, respectively, to the change of a single one.

Shannon (1948)'s definition of information only interprets the members of the ensemble as messages:

The significant aspect is that the actual message is one selected from a set of possible messages. The system must be designed to operate for each possible selection, not just the one which will actually be chosen since this is unknown at the time of design (Shannon 1948: 379).

The especially valuable approach demonstrates that a common and even mathematical concept underlies both an area addressing technics and another, studying nature. A bridge is outlined between the natural and artificial realm.

Similar ideas seem to be "floating in the air" in MIT then after Shannon's colleague Norbert Wiener (1948) had published the famous subsequently Cybernetics: Or Control and Communication in the Animal and the Machine.

The present paper extends the same "cybernetic synthesis" to the foundation both of nature (quantum mechanics) and mathematics (set theory) complementing a neo-Pythagorean nuance to that impressing and generalizing science such as cybernetics, or theory of information.

One can add that entropy and therefore information can be interpreted as a particular case of expectation, just that of the expectation of a probability distribution.

"Quantum information": Though information as above can be defined for any real functions, generalized to complex ones, and even so on, all this remains mainly a mathematical exercise for all those generalizations are not necessary for the solutions of experimental or empirical, scientific or technical problems.

However, the generalization of information, to which quantum mechanics is forced in order to be able to be reformulated in terms of information, is necessary for the solution of its basic problem: how to be unified and then uniformly described quantum leaps, i.e. discrete morphisms, and classical motions, i.e. smooth morphism differentiating from each other by the availability of velocity in any point of the trajectory in the latter case.
The solution turns out to involve necessarily infinity for it is impossible for any finite mathematical structure. So, the complex Hilbert space, which just underlies quantum mechanics, is not only infinitely dimensional in general, but furthermore just being complex, it requires a continuum to be defined even for any finite set of dimensions.

Thus quantum information introduced by quantum mechanics is not only a mathematical puzzle (in fact, not too difficult), but necessarily referring to infinity to resolve its problem:

Quantum mechanics turns out to be the first and probably single experimental science utilizing infinity necessarily therefore being in condition to make experiments on infinity in final analysis.

Accordingly, quantum information can be equivalently interpreted as the quantity of choices, each of which is among an infinite set of alternatives in general.

The generalization from classical to quantum information by their corresponding units of elementary choice, from a bit (as a "finite choice") to a qubit (as an "infinite choice"), is necessary for the solution of the main problem of quantum mechanics.

"Information as a binary series": Kolmogorov (1965) offered a fundamentally different understanding of information and then a new quantitative definition turned out to be equivalent to the entropic one in some degree (Kolmogorov 1968).

If information is some relation of orders or orderings, it can be realized as the reordering from one to other(s). The reordering is an algorithm as an unambiguous way to be constructed the latter(s) starting from the former, which is the "initial conditions" of the problem.

As the starting point can be also the "absolute chaos and disorder" or the "homogeneous chaos" (Wiener 1938) shared by all possible orders as a common "reference frame", any order should correspond one-to-one to some algorithm, which is able to create just this order from that "absolute chaos".

Further, any algorithm is equivalent to some Turing machine, which in turn can be unambiguously represented as a binary series, supposedly finite for the corresponding algorithm and Turing machine are to be finite according to the common sense for "calculation".

However, if quantum information refers to infinity in the sense above, the Kolmogorov definition seems to be to be generalized both to transfinite sequences and to series of transfinite ordinal numbers. However, there is not an obvious way for that meaningful generalization at first glance.

The main statement of this paper will attempt to resolve the problem about that generalization.

It is worth to add that the Kolmogorov definition interprets the concept of order as a well-ordering in the rigorous mathematical sense of the latter term.

"Choice": Though both information and quantum information can be understood and defined as a relevant mathematical relation of orders, they can be equivalently represented as the quantities of elementary choices, correspondingly bits and qubits.

"Choice" turns out to be more rigorous and precisely determinable concept and thus more relevant as the ground of information than "order".

Even more, both entropic and Kolmogorov definitions of information transform the concept of (dis)order in that of choice, each in a different way: 
The entropic definition uses the meditation of "probability" for the "probability" can be interpreted as the ratio of the choices of favorable alternatives to the choices of all alternatives instead of the ratio of the favorable ones to all.

The Kolmogorov definition is equivalent to a series of Turing sells, in which either " 1 " or " 0 " can be recorded.

Thus, the collection of an empty cell and both alternatives recordable in it is an elementary choice, a bit of information.

"Choice" and "well-ordering" are closely related and even equivalent to each other in a mathematical sense. Thus, the transition from "order" to "choice" means a transition to the duality of "(dis)order" and "well-ordering" in final analysis.

"Choice as the "atom" of the course of time": Though "choice" can be specified in an abstract way absolutely independent of "time", it can also be interpreted as an "atom" of time unifying the minimally possible "amount" of future, present, and past.

For example, the future is symbolized by the "empty cell" of the choice, the present by the action itself of choice, and the past by the chosen alternatives.

One can add that the choice as to the physical course of time is "infinite" for quantum information measurable by units of "infinite choice" is what underlies nature.

"Both quantum information and information as the quantity of choices": Indeed, the only difference between classical and quantum information consists in the units of measurement, correspondingly bits and qubits.

"The axiom of choice": Once the choice is related to infinity, one needs the axiom of choice for it to be guaranteed even in the cases where any constructive way to be made the choice is not known or cannot exist in principle.

Some constructive way to be made a choice among the elements of any finite set exists always.

The axiom of choice divides constructiveness from choice as to infinity, but relates the choice among any set whether infinite or finite.

Thus one can see the sense of the axiom so:

The choice from finiteness to infinity can be generalized at the expense of the loss of constructiveness, which loss in turn can be interpreted as "randomness".

"A choice from infinity": The axiom of choice has a series of "ridiculous corollaries" just as quantum mechanics implies not less "ridiculous" ones, though. Of course, this is hardly able to make the link between them more convincing:

One of those "peculiarities" is the statement that any (incl. infinite) set of choices from infinity is equivalent to a single one just as any (incl. infinite) set of qubits is equivalent to a single one.

The complex "Hilbert space" is the basic mathematical structure underlying quantum mechanics. It possesses a series of remarkable properties, a few of which are enumerated bellow:

1. It synthesizes arithmetic and geometry, positive integers and Euclidean space, which is the usual three-dimensional space of our experience.

2. Its elements are solution of the main problem of quantum mechanics: how to be united and described uniformly quantum leaps and smooth trajectories.

3. Its elements can be interpreted both as future states and as actual states of some quantum system
4. It coincides with the space dual to it. Thus the elements of the dual space are identical but complement to those of the initial Hilbert space.

5. Though the description by the complex Hilbert space needs only the "half" of variables in comparison to classical mechanics, any "hidden variables" cannot be added in principle. This mathematical structure possesses the rather extraordinary property to be inherently complete.

"Wave function" is term coined quantum mechanics to designate any element ("point") of Hilbert space as a state of some quantum system. The meaning of the term is easily to be extended to any interpretations of the complex Hilbert space and its elements such as the quantity featuring each infinite set unambiguously or as the values of quantum information.

That extended meaning of "wave function" addresses a certain ontological conclusion relating nature, mathematical structures, and information.

"Coherent state as an equivalent of a statistical ensemble": The designated as " 3 " property of Hilbert space above implies that any coherent state interpreted as a future one is equivalent to the corresponding collection of all present already measured states of the system.

"Quantum mechanics as a theory of quantum information": Quantum mechanics can be exhaustedly rewritten in the language of quantum information.

This means that the base of nature is only information:

There is nothing in the world besides quantum information!

Consequently, the general viewpoint in the paper can be featured as an information one:

Information is a universal and fundamental base in a series of scientific fields as the quantity of choices.

Choice underlies both physics as the "atom" of the course of time and mathematics as the "atom" of any mapping.

It is furthermore a "tool" for infinity to be described in detail quantitatively rather than qualitatively. In practice, this means that any infinite set can be distinguished from any other by its "wave function", which is a much more precise and rigorous description than the usual set-theory one by its ordinal and cardinal number. One can say that quantity of quantum information is what can differentiate the infinite sets from each other for the "wave functions" are the different values of it.

The main problem is how to be mathematically linked to each other unambiguously: (1) the new description of any infinite set by a value of the quantity of quantum information, which is a wave function, and (2) the usual set-theory description by an ordinal number being or representable as a transfinite binary series in general.

\section{THE METAPHYSICS OF QUANTUM INFORMATION}

The main statement: Quantum information is equivalent to the generalization of information from finite to infinite series.

There at least four fields of interpretation of the main statement: set theory, the foundation of mathematics, information theory, and quantum mechanics.

They will be considered one by one in detail:

Set theory can be interpreted as a mathematical doctrine of infinity. The concept of infinity is its core. However the quantities assigned to infinity such as ordinal and cardinal numbers are rather unspecified and not able to feature 
distinguishably an infinite set from another. For this purpose, any method for ascribing an exactly determined function to any infinite set is especially valuable.

The main statement above means just this:

Hilbert space and all "points" in it can be assigned to infinity as a set of discerning "names" for all infinite sets.

This is a method for the doctrine of infinity in mathematics to be transformed from a rather qualitative into a rigorous quantitative theory directly applicable in a series of areas.

Indeed the complex Hilbert space can be seen as an immediate generalization of Peano's arithmetic as follows:

Hilbert space is a free variable of quantum information as a well-ordered series of empty qubits. Any point in it (i.e. any "wave function") is a value of it therefore transforming it into a bound variable.

The $\mathrm{N}^{\text {th }}$ qubit can be interpreted as a "geometrical" generalization of the positive integer $\mathrm{N}$ after substituting the point "N" with the unit ball "N". Thus all values of this qubit "N" corresponds to the single point "N" after the "arithmetical degeneration" collapsing the qubit into a point.

Consequently, arithmetic and geometry turn out to be inherently linked in Hilbert space for it can be furthermore considered as an infinite-dimensional generalization of the usual Euclidean three-dimensional geometry:

From the "viewpoint of arithmetic", the geometrical dimension of Hilbert space should refer to infinity describing exhaustedly any infinite set in a unique and relevant way.

From the "viewpoint of geometry", the arithmetical dimension of Hilbert space is a degeneration from unit balls to points or the borderline case of a limit where the magnitude of the "unit" converges to zero.

Both viewpoints can be unified into a single information one by the concept of choice as follows: $\mathrm{T}$

The geometrical viewpoint is "before" the choice, and the arithmetic one, "after" it. Information and more precisely quantum information mean the quantity of those choices, each of which is a single geometrical and arithmetical unit.

Both arithmetical and geometrical dimension share infinity but in different ways in a sense:

The arithmetical dimension implies rather the potential infinite of an infinite constructive process "adding and adding units, one by one": the "principle of (transfinite) induction".

The geometrical dimension implies rather the actual infinite as the result of the above process as the limit of converging into a single point.

Indeed the concept of actual infinity means just this: the entire constructive process of the potential infinity to be "reduced" to its whole and eventually named as a single point in Hilbert space, a.k.a. the result, to which converges the potential constructive process into infinity.

The same property to link inherently potential and actual infinity can be deduced also from the "duality to the axiom of choice" and therefore, to the inherent duality of Hilbert space.

The next step to the foundation of mathematics originates immediately from the previous interpretation in terms of set theory for the latter is the standard base of all mathematics unfortunately attacked by the "Gödel incompleteness theorems" (Gödel 1931).

One should search for that set, which is relevant to completeness for the granted one (e.g. that of real numbers in the case of the main theorem of algebra) can turn out to be unsufficient.

Thus, arithmetic granted to be the necessary and self-obvious base for set theory and via it, to all mathematics is unsufficient as the Gödel theorems demonstrate. One can search for that set, possibly including arithmetic, which is indeed relevant, independently of the prejudice:

Right the complex Hilbert space by its properties both to unify geometry and arithmetic and to name infinity in detail is a relevant applicant for the base of the cherished completeness and therefore, of the self-foundation of all mathematics.

The theorems about the absence of hidden variables in quantum mechanics (Neumann 1932; Kochen and Specker 1968) show not only that it is complete, but furthermore that Hilbert space, which is a sufficient premise for the theorems, implies that completeness by itself. One can say that the complex Hilbert space is inherently a complete mathematical structure.

In fact, infinity in the set theory grounded on arithmetic remains a too undetermined concept accumulating contradictions without distinguishing discernibly an infinite set from any other of the same power. The concept of infinity though being exceptionally fruitful is what opens the "backdoor" of mathematics to contradictions.

If the infinite sets are reliable differentiated from each other by their "wave function", this does not allow of identifying different infinities being due to the uncertainty of the term "infinity", and thus of sneaking contradictions through the holes of a fussy concept.

The idea for the Gödel incompleteness to be overcome is the following:

If one considers the Gödel number of the so-called first incompleteness theorem ("Satz VI", Gödel 1931: 187), it turns out to be infinite necessarily because its statement includes all positive integers explicitly. Consequently, its negation will share the "same" infinite number. Thus, the theorem is presupposed the incompleteness, which it will prove by its proof.

One can admit that special kind of statement - "liar" stating the own unprovability and fundamental for the Gödel theorems forces the theorem proved by its assistance to share an inherent uncertainty.

That kind of incompleteness or uncertainty can be avoided by ascribing a different wave function for any meta-statement possessing equal right one and the same infinite number just as their negation.

One can also complement that the completeness of Hilbert space, being inherently intrinsic, addresses some kind of neoPythagorean ontology.

Quantum mechanics as a doctrine about nature and underlain right by Hilbert space is also evidence of the same kind of mathematical ontology.

Information theory is the next area of interpretation. Information as a quantity can be seen at least in two quite different ways: (1) as some kind of relations between two real functions (e.g. as the functional of the one in the basis of the other) interpretable as the entropies of any two states of a single system or as those of two systems; and (2) as some binary series interpretable both as a Turing machine and as an algorithm able to transform the one of the above states or systems into the other.

A few comments seem to be necessary:

1. The mathematical form of entropy is identical to that of expectation (representing a particular case of expectation; the 
expectation of the probability distribution itself). Consequently, entropy can be interpreted in two ways sharing one and the same formula and even mathematical structure: (1) as the entropy (in a proper sense) of a real state of some statistical ensemble; (2) as the expectation of the same real state.

The probabilities will be "objective" in the former case and "subjective" otherwise. However, as being "subjective" as being "objective" is not more than a different interpretation of one and the some formula therefore hinting some hidden common "state of affairs" underlying both cases.

Hilbert space is what generalizes that twofold interpretation as to any element of it.

The concept of information thus allows of a common quantity representing a relation of entropy (in a proper sense) and expectation. This means a quantity shared by present and future states (as an example).

2. One can tempt to add the past to the range of information. This can happen if the past is axiomatized mathematically as well-ordering and therefore as some number series reducible to binary.

3. Summarizing the above two considerations, one can conclude that the main statement interpreted in terms of information theory means generalizing the concept of information also to the past extending the invariance of the description of entropy and expectation as to well-ordered number series. One can simply say:

Information is invariant to time, to past, present, and future, therefore being one of the most fundamental quantities, both physical and mathematical.

4. The main statement can be also interpreted as that generalization, which extends the range of the equivalence of Kolmogorov information and entropic information from finite to infinite series and statistical ensembles

4.1. Defined as a Turing machine or as an arbitrary algorithm, information can be immediately referred only to finite series.

4.2. Defined as entropic information, it addresses implicitly also only finite statistical ensembles nevertheless that the approximating real functions refer to the continuum, on which they are determined. That continual infinity is not inherent to the finite statistical ensemble, to which entropic information refers in fact.

4.3. Unlike these "classical cases", both quantum information and transfinite ordinals are directly related to infinite.

This is obvious even in definition as to the transfinite ordinals.

Quantum information being a quantity of qubits addresses also inherently infinity as soon as a qubit can be interpreted as a choice among the elements of an infinite set.

Quantum mechanics being the "fatherland" of quantum information allows of interpreting its main equation, the Schrödinger equation, as a physical exemplification of the main statement:

We suggest that the Schrödinger equation is well-known in all modifications. So, it will be sufficient and more suitable to describe it only qualitatively for the present objectivity:

It equates the first time derivative (therefore the change in time) of the wave function (therefore a value of quantum information) of any quantum system to the algebraic sum of (1, with sign minus sign) the second space derivative (laplacian and thus the tensor product of two changes in space) of the same wave function (and the same value of quantum information) corresponding to kinetic energy in classical mechanics and of (2, with a plus sign) the product of a potential function and the same wave function, corresponding to potential energy in classical mechanics.

Both sides of the equation have the physical dimension of energy. The coefficients involved in the equation are the Planck constant, the mass of the system (more exactly, the reduced mass of the system), and the imaginary unit, $i$, which is very important for the information interpretation of the equation.

A few preliminary comments are necessary:

1. The Schrödinger equation is a generalization of the fundamental law of energy conservation in classical mechanics: Indeed the former implies the latter if the wave function is a constant as a particular case.

In turn, the generalization involves just complex functions being inapplicable as to any real functions. One may suggests then that quantum information corresponds to a relevant generalization of energy already definable as to complex values therefore involving a second, i.e. just imaginary dimension of energy. That dimension is interpretable as "time energy" unlike kinetic and potential energy both "space energies".

Unfortunately the suitable term of "space-time energy" as equivalent to quantum information would call a series of messes with special and general relativity.

Another option for them is to be correspondingly designated as temporal and spatial energy. Then, one of the possible meanings of the Schrödinger equation would be their equating. Furthermore, the spatial energy would correspond to quantum information defined as a statistical ensemble, and the temporal energy to that defined as a transfinite ordinal

2 . The potential and kinetic energy are clearly distinguished from each other in classical mechanics: Kinetic energy corresponds to the real motion depending only on its velocity, and potential energy describes the force in each spatial point acting on a tentative reference unit (e.g. a body or a material point) depending only on its position. The law of energy conservation means the way, in which the acting of the force is transformed into the motion of some real item. The Schrödinger equation generalizes the classical understanding as follows:

The wave function corresponds to space in a different way in the cases of kinetic and potential energy: The tensor product of two identical and complementary (or "conjugate") wave functions is mapped into space if the energy is kinetic, and a single wave function is mapped into space in the case of potential energy. Indeed the latter can be interpreted as the action on an energy-momentum unit thus depending only on the spatial coordinates.

However this representation in the Schrödinger equation, which is closer to the ideas of classical mechanics, is "paraphrased" at present (and particularly, in the Standard model) as a few kinds of fundamental symmetries: any symmetry is a relation between a set of symmetric elements as different and the same set as a single element being a class of equivalence to the symmetry in question.

Thus, the concept of symmetry expresses differently but equivalently the underlying idea of quantum information: the choice of an element among a set of equivalent, but different alternatives.

Nevertheless the form of the Schrödinger equation is universal as it refers to all possible symmetries rather than to the 
ones really observed in experiments until now or to the ones included in the Standard model.

3. The invariance of discrete leaps and smooth motions allows of the Schrödinger equation to be represented by the corresponding units: bits and qubits.

Furthermore, one can introduce a new distinction between real (or kinetic) and potential qubits: the formers for kinetic energy, the latters for potential energy.

Then, the Schrödinger equation can be formulated so:

The energy of a "(trans)finite bit" is equal to the subtraction of the energy of a potential and a kinetic qubit.

The necessary equivalence of potential and kinetic qubits is implied by the equivalence of the probabilistic (Born 1927) and many-worlds interpretation (Everett III, 1957) and in turn implies the equivalence of a bit of a transfinite position ("transfinite bit") and a bit of a finite position ("finite bit").

4. The Kochen - Specker (1968) theorem helps us to avoid a mess:

The equation of the energies as above does not mean that a qubit can be equivalent to a bit, which would contradict to the direct corollary (Kochen, Specker 1968: 70), which is immediately after the main theorem of their paper (ibid., a few lines above).

In fact, the concept of energy conservation (only generalized in the Schrödinger equation), as Emmy Noether's (1918) fundamental theorem shows, implies the equality of all moments of time.

Consequently, the equation of energies implies that the past moment corresponding to a bit, the present moment: to a kinetic qubit, and the future moment: to a potential qubit are already presupposed as equivalent, but this does not refer to the bits and qubits themselves: They are fundamentally different, and only the quantity of their corresponding energy can be equated.

Summarizing all consideration above, one can suggest the following meaning of the Schrödinger equation in terms of quantum information:

It generalizes energy conservation to past, present, and future moments of time rather than only to present and future moments as this does the analogical law in classical mechanics.

Thus, it is the universal law of how "time flows" or in other words, about the course of time.

Furthermore, resolving its problem, the Schrödinger equation suggests the proportionality (or even equality if the units are relevantly chosen) of the quantities of both quantum and classical information and energy therefore being a (quantum) information analogy of Einstein's famous equality of mass and energy (" $\left.E=m c^{2} "\right)$.

\section{CONCLUSIONS}

This paper shows how existing concepts in a few scientific areas can be synthesized by a single statement. Its proof is technically almost trivial, and thus it interested from a philosophical rather than properly mathematical viewpoint.

The main statement establishes the link between a transfinite number assignable to some infinite set and an element of Hilbert space interpretable both as a "wave function" in terms of quantum mechanics and a given value of quantum information.

It allows furthermore of synthesizing at least four quite different scientific fields (set theory, the foundation of mathematics, information theory, and quantum mechanics): All of them to be considered as four interpretations of a single underlying mathematical structure, that of the complex Hilbert space.

That synthesis in turn addresses far-reaching speculations about ontology, epistemology, and other branches of philosophy for the following reason:

Quantum mechanics is a fundamental and scientific doctrine about nature; set theory and the foundation of mathematics underlie mathematics; and information theory is implemented in an essential part of the contemporary technics.

If there is a "bridge" for direct, one-to-one interpretation between them, this implies some kind of neo-Pythagorean ontology making related mathematics, physics, and technics immediately, by an explicit mathematical structure.

Number and being (by the meditation of time), the natural and artificial turn out to be not more than different hypostases of a single common essence.

\section{REFERENCES}

Aspect, A., Grangier, R., Roger, G. (1981) "Experimental Tests of Realistic Local Theories via Bell's Theorem", Physical Review Letters, 47(7): 460-463.

Aspect, A., Grangier, R., Roger, G. (1982) "Experimental Realization of Einstein-Podolsky-Rosen-Bohm Gedanken Experiment: A New Violation of Bell's Inequalities", Physical Review Letters, 49(2): 91-94. Bell, J. (1964) "On the Einstein - Podolsky - Rosen paradox", Physics (New York), 1(3): 195-200.

Born, M. (1927) "Physical aspects of quantum mechanics," Nature 119: 354-357.

Cantor, G. (1897) "Beitrage zur Begrundung der transfiniten Mengenlehre (Zweiter Artikel)", Mathematische Annalen, 49(2): $207-$ 246

Einstein, A., Podolsky, B., Rosen, N. (1935) "Can Quantum-Mechanica Description of Physical Reality Be Considered Complete?" Physical Review, 47(10): 777-780.

Everett III, H. (1957) „Relative state” Formulation of Quantum Mechanics," Reviews of Modern Physics 29(3): 454-462.

Gödel, K. (1931) "Über formal unentscheidbare Sätze der Principia mathematica und verwandter Systeme I," Monatshefte der Mathematik und Physik 38(1): 173-198.

Kochen, S., Specker, E. (1968) "The problem of hidden variables in quantum mechanics", Journal of Mathematics and Mechanics, 17(1): 5987.

Kolmogorov, A. (1965) "Tri podkhoda k opredeleniyu "kolichestvo informatsii" (Three approaches to the definition of the quantity of information), Probl. Inf. Transm. 1: 3-11

Kolmogorov, A. (1968) "Logical basis for information theory and probability theory," IEEE T. Inform. Theory IT-14: 662-664.

Neumann, J. von (1923) "Zur Einführung der trasfiniten Zahlen", Acta litterarum ac scientiarum Ragiae Universitatis Hungaricae FranciscoJosephinae, Sectio scientiarum mathematicarum, 1(4): 199-208

Neumann, J. von (1932). Mathematical foundation of quantum mechanics [Mathematische Grundlagen der Quantenmechanik, Berlin: Springer, pp. 167-173 (Chapter IV.2).

Noether, E. (1918) "Invariante Variationsprobleme," Nachr. d. König. Gesellsch. d. Wiss. zu Göttingen, Math-phys. Klasse 1918, 235-257.

Russell, B, Whitehead, A. N. (any edition) Principia Mathematica, Vol. $2(* 153)$, Vol. $3(* 251)$.

Schrödinger, E (1935) "Die gegenwärtige situation in der Quantenmechanik", Die Naturwissenschaften, 23(48), 807-812; 23(49), 823-828, 23(50), 844-849.

Shannon, C. (1948) "A Mathematical Theory of Communication," Bell System Technical Journal, 27 (3), 379-423; 27 (4), 623-656.

Wiener, N. (1938) "The Homogeneous Chaos," American Journal of Mathematics, 60 (4), 897-936.

Wiener, N. (1948) Cybernetics; or, Control and communication in the animal and the machine, Cambridge, Mass.: Technology Press. 